INPLASY

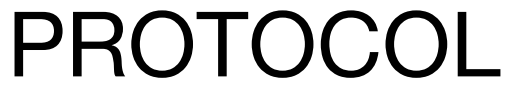

To cite: Bashir et al. Effects of Physical Trainings on Skillrelated Fitness Components of Kabaddi Players: A Systematic Review. Inplasy protocol 2021100050. doi:

10.37766/inplasy2021.10.0050

Received: 16 October 2021

Published: 16 October 2021

Corresponding author: Marrium Bashir

gs61096@studnt.upm.edu.my

Author Affiliation:

Universiti Putra Malaysia

Support: Self finance.

Review Stage at time of this submission: Preliminary searches.

Conflicts of interest:

None declared.

\section{Effects of Physical Trainings on Skill- related Fitness Components of Kabaddi Players: A Systematic Review}

\author{
Bashir, M1; Geok, SK²; Akbar, S3; Raza, A4 .
}

Review question / Objective: The objective of this to assess the effects of physical trainings on skill-related fitness components of kabaddi players.

Condition being studied: Physical trainings on skill-related components in kabaddi players.

Eligibility criteria: All types of physical trainings on aspect of skill-related fitness of athletes were included, Criteria for inclusion of studies consisted of: (1) published in English full articles, describing the use of athletes (male and female); (2) conducted among kabaddi players aged 17-28 years; (3) studies involved all types of physical trainings or combinations of physical trainings and exercises training interventions; (4) Skill-related fitness components (speed, power, reaction time, agility, balance, coordination); (5) randomized controlled trial (RCT), non-randomized controlled trial (Non-RCT) with two or more groups, and single-group trials with pretest and post-test design; (6) published review articles, meta-analysis, validation studies.

INPLASY registration number: This protocol was registered with the International Platform of Registered Systematic Review and Meta-Analysis Protocols (INPLASY) on 16 October 2021 and was last updated on 16 October 2021 (registration number INPLASY2021100050).

\section{INTRODUCTION}

Review question / Objective: The objective of this to assess the effects of physical trainings on skill-related fitness components of kabaddi players.
Condition being studied: Physical trainings on skill-related components in kabaddi players.

\section{METHODS}

Search strategy: Search for articles published in English in the following databases: PubMed, EBSCOHOST, WOS, 
Scopus and in additional References, Google Scholar. In each database, a search was conducted by title; taking a predefined combination of keywords used included: "skill-related components", "skill related components", "skill-related fitness components", "skill related fitness components", "fitness components", "Skillrelated fitness", "skill related fitness", "speed", "power", "agility", "quickness", "balance", "coordination", "reaction time", "physical trainings", "physical exercises", "kabaddi", "Kabaddi players" and "kabaddi athletes". These keywords were used separately and in combination.

Participant or population: Male and female kabaddi players.

Intervention: Physical trainings.

Comparator: Single-group trials and two or more groups.

Study designs to be included: RCT or NonRCT.

Eligibility criteria: All types of physical trainings on aspect of skill-related fitness of athletes were included, Criteria for inclusion of studies consisted of: (1) published in English full articles, describing the use of athletes (male and female); (2) conducted among kabaddi players aged 17-28 years; (3) studies involved all types of physical trainings or combinations of physical trainings and exercises training interventions; (4) Skill-related fitness components (speed, power, reaction time, agility, balance, coordination); (5) randomized controlled trial (RCT), nonrandomized controlled trial (Non-RCT) with two or more groups, and single-group trials with pretest and post-test design; (6) published review articles, meta-analysis, validation studies.

Information sources: PubMed, EBSCOHOST, WOS, Scopus, Google and Google Scholars.

Main outcome(s): Skill-related fitness components (speed, power, reaction time, agility, balance, coordination.
Quality assessment / Risk of bias analysis: Not reported.

Strategy of data synthesis: Not reported.

Subgroup analysis: Not reported.

Sensitivity analysis: Not reported.

Language: English.

Country(ies) involved: Pakistan, Malaysia.

Keywords: Physical trainings, Skill related components, Kabaddi players.

Contributions of each author:

Author 1 - Marrium Bashir.

Email: gs61096@student.upm.edu.my

Author 2 - Soh Kim Geok.

Email: kims@upm.edu.my

Author 3 - Saddam Akbar.

Email: justsaddamakbar4@gmail.com

Author 4 - Ali Raza.

Email: aliraza09055@gmail.com 